\title{
Docentes investigadores de programas de Pós-graduação em educação no Brasil: estudo sobre o uso de recursos informáticos no processo de pesquisa
}

\author{
Ricardo Antonio Gonçalves Teixeira \\ Francislê Neri de Souza \\ Rui Marques Vieira
}

Resumo: Este estudo propõe compreender o perfil dos docentes brasileiros em relação ao uso de recursos tecnológicos, mais especificamente os computacionais, no âmbito de suas investigações. Para eleger os sujeitos da pesquisa, optou-se por inquirir, a partir da aplicação de questionários, um grupo representativo de investigadores da área de Educação ligados a programas de Pós-Graduação em nível de mestrado e doutorado de diferentes Instituições de Ensino Superior, referenciadas pelo Ministério da Educação do Brasil como programas de excelência. A base de análise partiu da abordagem quantitativa em um estudo de caráter exploratório a partir de multiplicidades de dimensões de conteúdos presentes nos posicionamentos e ideias dos sujeitos pesquisados. Como orientação dos procedimentos metodológicos buscou-se na literatura as bases e fundamentos epistemológicos acerca de princípios e processos tecnológicos, a partir de um contexto histórico, bem como documentos oficiais para fundamentação política. Como principais resultados, foi possível identificar concentração dos programas de mestrados e doutorados em Educação, de excelência, na Região Sudeste do Brasil; presença de novos docentes, em tempo no programa e em idade; forte correlação entre a idade dos docentes inqueridos com o período de formação em nível de graduação, com correlação reduzida em nível de mestrado e não confirmada em nível de doutorado; utilização de bases eletrônicas de dados pela maioria absoluta dos docentes pesquisados, e de softwares no âmbito de suas pesquisas, valendo-se de pesquisas mistas (quali-quantitativa), com apoio de recursos informatizados.

Palavras-chave: Perfil docente. Programas e Pós-Graduação em educação. Processos de pesquisa. Software de apoio à análise de dados.

\section{Researchers teachers of graduate programs in education in Brazil:}

a study on the use of computer resources in the research process

Abstract: This study aims to understand the profile of Brazilian teachers regarding the use of technological resources, specifically computer as part of their investigations. To elect the subjects, it was decided to inquire from the questionnaires, a representative group of researchers in the field of Education related to graduate programs at Masters level and Ph.D. from different Higher Education Institutions, referenced by the Ministry of Education of Brazil as excellent programs. The basis of the quantitative analysis was based approach in a study of exploratory from the multiplicities of the dimensions of content present in the positions and ideas of the subjects studied. As a guide the methodological procedures sought in the literature and epistemological bases about principles and technological processes, from a historical context, as well as official documents for political reasons. As main results, we could identify concentrations of masters and doctoral programs in Education, excellence, in southeastern Brazil; presence of new professors in the program time and age; strong correlation between the age of the surveyed teachers with the training period at the undergraduate level, with reduced level of uncommitted master and doctoral-level correlation; use of electronic databases by the absolute majority of teachers surveyed, and software within their research, making use of (qualitative and quantitative) joint research with the support of computer resources.

Key words: Teacher Profile. Programs and Graduate Education. Research processes. Software to support data analysis. 


\section{Introdução}

Muitas são as técnicas, recursos, meios, procedimentos, modalidades, abordagens e métodos adotados em diferentes pesquisas, por diferentes pesquisadores em todo o mundo, com finalidades distintas. Conhecer os aportes teórico-metodológicos utilizados e compreender a complexidade dos procedimentos de investigação se constitui como centro desta contribuição científica.

Todos reconhecemos que as tecnologias em geral e o computador ligado à internet, em particular, causaram e têm causado uma verdadeira revolução na forma como os pesquisadores em educação têm conduzido as suas investigações. No entanto, existem indícios de que nem sempre as ferramentas adequadas a cada processo de pesquisa são devidamente utilizadas. Por isso, nunca é demais repetir a advertência feita há mais de 15 anos: "The researcher who does not use software beyond a word processor will be hampered in comparison to those who do" (MILES; HUBERMAN, 1994, p. 44).

Neste sentido, o presente trabalho se justifica pela necessidade de compreender os principais procedimentos de pesquisa adotados por pesquisadores brasileiros, bem como verificar o uso que fazem de recursos informatizados na condução de suas investigações.

Igualmente, esta pesquisa é relevante por mapear as principais metodologias, métodos e procedimentos de análise (finalidade, objetivo e abordagem) estabelecidos nas pesquisas, além da identificação das principais dificuldades e desafios técnicos e metodológicos que os pesquisadores enfrentam nas diferentes fases de suas pesquisas.

O presente artigo é parte de um projeto de pesquisa interinstitucional sobre o uso de recursos informáticos por investigadores em suas pesquisas, e se propõe a apresentar um estudo sobre o perfil tecnológico de docentes pesquisadores de programas de pós-graduação em educação de excelência no Brasil. Buscou-se, neste sentido, registrar os diferentes posicionamentos dos pesquisadores frente ao uso de recursos informatizados nas situações de investigação.

São sujeitos desta pesquisa investigadores brasileiros de diferentes instituições contemplados com a Bolsa Produtividade da área de Educação concedidas pelo Conselho Nacional de Desenvolvimento Científico e Tecnológico (CNPq), com vigência até o ano de $2016^{1}$. No entanto, a partir de tendências internacionais de trabalhos colaborativos e investimentos brasileiros para pesquisas com

1 Para mais informações acesse o endereço eletrônico: <http://plsql1.cnpq.br/divulg/RESULTADO_ PQ_102003.curso> 
este fim, optamos por eleger, como sujeitos do estudo, não os investigadores de forma isolada, mas os participantes de programas de mestrados e doutorados enquadrados pelo Ministério da Educação do Brasil (MEC) como programas de excelência, ou seja, aqueles programas que, na avaliação da Coordenação de Aperfeiçoamento de Pessoal de Nível Superior (CAPES), receberam nota 4 ou $5^{2}$. Assim, a nosso ver, estaríamos contemplando o padrão de qualidade almejado em pesquisa.

A pesquisa traz, no enquadramento teórico, uma abordagem histórica sobre a questão de ciência e método, bem como reflexões acerca de abordagens quantitativas e qualitativas e seus reflexos nas pesquisas. Traz para o âmbito da discussão, o uso de recursos tecnológicos, enfocando, mais especificamente, a utilização de recursos informatizados como apoio e suporte às pesquisas. Apresenta o processo evolutivo de softwares de apoio a análises quantitativas (a partir da década de 1960) e qualitativas (a partir da década de 1980), e discute a possibilidade de integração, em termos de uso e aplicação, de pesquisas com abordagem mista (quali-quantitativas).

\section{Enquadramento teórico}

Mesmo após séculos de fervorosos debates e discussões acerca de métodos de investigação, ainda hoje, a abordagem adotada na pesquisa causa polêmica entre pesquisadores de diferentes áreas de conhecimento. Afinal, que tipo de abordagem nos conduz a uma melhor análise dos fatos, proporciona melhores condições e, consequentemente, melhores resultados ao estudo realizado: a abordagem quantitativa, qualitativa ou uma abordagem híbrida, ou seja, mista? Quais as principais marcas, acentuadas em diferenças e similaridades entre elas? Que áreas do conhecimento ou segmentos de pesquisadores se utilizam de uma ou outra abordagem? Quais são as suas peculiaridades epistemológicas? Em decorrência de tais questões, é igualmente importante salientar a postura do pesquisador frente a uma ou a outra natureza de investigação, assim como o papel das tecnologias informatizadas neste processo.

A ciência moderna, assentada na razão positivista, tem se ocupado em processos de exploração da observação, experimentação, comparação e classificação que se firmaram como método válido para obtenção de dados con-

2 A CAPES é uma fundação do Ministério da Educação (MEC) que desempenha papel fundamental na expansão e consolidação da pós-graduação stricto sensu (mestrado e doutorado) em todos os estados da Federação. Os programas de mestrado e doutorado são avaliados de três em três anos sendo pontuados segundo uma escala de sete pontos, sendo considerados de excelência os programas com notas igual ou superior a cinco. 
fiáveis ("reais"). Na base deste construto se encontra a matemática, poderoso instrumento que possibilita à ciência a busca e consolidação da "verdade". Assim, a base quantitativa de análise que busca traduzir, estatisticamente, generalizações de resultados a partir de leis universais, tem sido a marca da ciência moderna para a explicação da realidade.

Historicamente arraigada em bases filosóficas e sociais, a ciência moderna, adentra ao fecundo campo da psicologia e seu desdobramento na área de educação. Esta nova ciência, a partir de 1915, conforme apresenta Franco (2008, p. 8), "define, em princípio, seu próprio objeto de estudo: o comportamento humano", rejeitando a noção intuitiva de homem em detrimento de uma abordagem dita mais objetiva e contextualizada.

Considerando-se que objetividade, como construto das "verdades", é uma marca sólida do modelo científico adotado e aceito nas academias, as pesquisas de cunho quantitativo, têm sido o modelo reconhecido pelos principais veículos de divulgação de pesquisas e exigido pelas agências de financiamentos.

A produção de conhecimento, fortemente influenciada pelo modelo capitalista de sociedade, é impulsionada, segundo Lima e Mioto (2007), pelo movimento articulado entre Universidade-Empresa-Estado, diminuindo sistematicamente o poder das academias e influenciando novos caminhos para as práticas de saberes. Orientadas pelas demandas e impulsionadas pelas novas tecnologias computacionais, as universidades tornam-se mais operacionais, no sentido de produtividade. Em decorrência deste fato, com apoio financeiro do Estado e de instituições privadas, as universidades passam a incentivar a pesquisa e a formar novos pesquisadores, no âmbito da pós-graduação, para atendimento às novas demandas.

Nesse ínterim, surgem os primeiros softwares e aplicativos, programas especializados na execução de determinadas tarefas, para apoio às pesquisas. Os primeiros softwares surgiram no final da década de 1950 e início da década de 1960, em atendimento às necessidades de análises estatísticas de pesquisadores e institutos de pesquisa. Este período, conforme apresenta Bardin (2010), é caracterizado pela expansão das aplicações de técnicas diversificadas em termos metodológicos. No ano de 1968, surge o Statistical Packcage for Social Sciences (SPSS), a primeira versão do software estatístico que se tornaria, pelo grande volume de acesso por pesquisadores, um dos softwares de apoio a pesquisas quantitativas mais utilizado em todo o mundo na área das ciências humanas e sociais.

A pretensa postura neutra do pesquisador frente ao objeto pesquisado, o princípio rígido no levantamento de amostras, as regras e procedimentos 
adotados em conformidade com as circunstâncias, o tratamento estatístico dos dados coletados como requisito para confirmar ou refutar as hipóteses levantadas e o construto de leis universais para explicação de fenômenos, são marcas gerais da abordagem quantitativa. Tal abordagem, embora com necessárias adaptações, se encontra presente em pesquisas de diversas áreas, tais como as ciências sociais e aplicadas, exatas e da terra, biológicas, da saúde, engenharias e, mais recentemente, como apresentado, as ciências humanas.

É certo que, embora se constitua como modelo padrão de pesquisa por diferentes áreas do saber, o método quantitativo não era único e suficiente para dar respostas aos diferentes problemas de ordem histórica, social, política e econômica. Mesmo estando à margem dos moldes exigidos pela academia, empresas e estado, a pesquisa qualitativa se apresentava como um movimento crescente nos EUA, na Europa e em alguns países da América Latina. Esta modalidade de pesquisa, segundo Denzin e Lincoln (2008), não é determinada exclusivamente pelo método ou técnica, mas pela forma como é trabalhada e concebida.

Diferentemente da pesquisa quantitativa, que apresenta um histórico de estabilização em termos de método e teorias, a pesquisa qualitativa não pode ser compreendida em um único movimento, presente em um determinado espaço temporal. Segundo Denzin e Lincoln (2008), ela é fruto de oito períodos, fases ou momentos históricos, tendo cada um distintas características. Junto às características, apresentam-se contextos sobre os softwares de apoio a pesquisas quanti e qualitativas disponíveis.

Período Tradicional (Traditional Period), compreendido entre o final do século XIX e o final dos anos de 1940, com o fim da II Guerra Mundial: neste período foram criados os primeiros protótipos de computadores: Colossos, por Alan Turing, uma máquina utilizada para resolver códigos secretos de guerra; Mark I, uma gigantesca máquina de calcular que efetuava cálculos complexos em tempo reduzido; ENIAC, um computador construído para calcular trajetórias de mísseis com precisão; EDSAC, conhecida como máquina de Von Neumann, o primeiro computador a usar fitas magnéticas.

Fase Modernista (Modernist Phase), de 1950 a 1970: os computadores, além de fins militares, passaram a ser utilizados, em grande parte do mundo, como elemento de apoio e suporte a pesquisas científicas nas universidades, ressaltando-se que nesse período, como apresentado, o software estatístico SPSS é desenvolvido.

Gêneros Indefinidos (Blurred Genres), período de 1970 a 1986: surgem as primeiras versões de softwares qualitativos como The Ethnograph, NUD*IST, 
Vivo, Atlas TI, dentre outros softwares de indexação, busca e teorização de dados não numéricos e não estruturados; no âmbito quantitativo, nesse período, o SPSS lança as primeiras versões, em DOS, para computadores portáteis, e inúmeros outros softwares quantitativos são lançados no mercado.

Crise da Representação (Crisis of Representation), a partir da metade dos anos de 1980 a 1990: tanto os softwares de análise qualitativa, quanto os de análise quantitativa continuam em processo de desenvolvimento, apresentando ao mercado outras versões com correções e avanços.

Crise Tripla (Triple Crisis), que compreende a década de 1990 a 2005, sintetizando os três momentos restantes da pesquisa qualitativa: surge, nesse período, a primeira versão do NUD*IST Vivo (NVivo), bem como o lançamento paralelo das versões NUD*IST 6 e o NVIVO 2; o SPSS lança as primeiras versões Windows e Macintosh.

E, mais recentemente, o oitavo momento, o da Repercussão Metodológica (Methodological Backlash), de 2005 aos dias atuais: na pesquisa qualitativa, é lançado o NVivo 7, com possibilidade de buscas e tratamento analítico; NVivo 8, nova geração de software com possibilidade de trabalhar com textos, áudios, vídeos e imagens; NVivo 9, que apresenta planilhas e banco de dados; NVivo 10, com implementação de mídias sociais e páginas da WEB; WebQDA, software que traz o elemento colaborativo mais efetivo e disponibilizado online; o SPSS lança diferentes versões, dentre elas a Survey, a Windows e a Mac.

A primeira das oito fases da pesquisa qualitativa, Período Tradicional, é caracterizada pela ação solitária de pesquisadores que, com suas próprias crenças, adotavam normas etnográficas clássicas, tratadas pelas autoras como mitos, de objetividade analítica, cumplicidade com o imperialismo e crenças no monumentalismo e na universalidade dos resultados.

A Fase Modernista, segundo momento, é marcada pelo surgimento de novas teorias interpretativas, tais como a etnometodologia, a fenomenologia, a teoria crítica e o feminismo. Nesse período, influenciados pelo paradigma pós-positivista, os pesquisadores tentaram aproximar a pesquisa qualitativa da quantitativa, propondo argumentos rigorosamente calcados nos paradigmas positivista e pós-positivista.

Retratado por Gênero Indefinido, esta fase é marcada pela maturidade da pesquisa qualitativa frente aos diferentes métodos, formatos, estratégias e teorias utilizados. Os computadores passam, neste momento, a serem utilizados como instrumento de apoio às pesquisas.

Na quarta fase, denominada Crise da Representação, é evidenciada a fragilidade do modelo antropológico de objetivismo, cumplicidade ao colonialismo 
e estruturalismo cultural e social. Surgem reflexões mais profundas sobre questões de raça, gênero e classe social.

A quinta, sexta e sétima fases, denominadas pelas autoras de Tripla Crise, são caracterizadas pela crise dos pesquisadores qualitativos em termos de representação, legitimidade e práxis. A quinta fase, é decorrente da constatação do abandono da figura do observador a distância, devido à impossibilidade de apreender as experiências vividas, pois, em essência, esta é induzida e consolidada pelo pesquisador, por meio de registros construídos socialmente. $\mathrm{Na}$ sexta fase, segunda das crises, é reascendida a legitimação dos teores científicos utilizados anteriormente: validade, generalidade e confiabilidade. Estas crises remontam a uma sétima fase, a da práxis, em que as ciências sociais e humanas se atentaram para questões de mudanças em um mundo em transformação.

A oitava fase, Metodologia de Backlash, retrata mais um momento de crise em que cientistas sociais, financiados pelo então presidente dos Estados Unidos da América, George W. Bush, formularam projetos e políticas encomendadas sobre problemas sociais como educação e pobreza, a partir de estruturas estritamente definidas como quantitativas, com base no pensamento positivista, interferindo, ideologicamente, nos resultados parciais apresentados em relatórios.

As relações contraditórias entre os aspectos quantitativos e qualitativos da pesquisa, como apresentado, são marcas históricas da construção de saberes científicos. Se, como apresenta Lefevre e Lefevre (2005), as abordagens quanti e quali se posicionaram, historicamente, em polos distintos, divergindo posturas e opiniões, as tecnologias da informação e comunicação, de forma oposta, apresentaram convergência quanto ao uso e aplicação, tornando-se um elemento, de certa forma, integrador.

Ao apresentar fortes críticas ao rígido modelo positivista da ciência, com bases teóricas e dogmáticas de séculos anteriores, Santos (2009) contrapõe-se a tal pensamento, refletindo sobre a mesma sociedade que experimenta potencialidades tecnológicas na comunicação, informação e interação para além do seu tempo.

As facilidades provocadas pelas tecnologias da informação e comunicação, dentre elas os aspectos comunicacionais e interativos, entendidas por Kenski (2007, p. 38) não apenas como suporte tecnológico, mas como "linguagens e maneiras particulares de comunicar-se com as capacidades perceptivas, emocionais, cognitivas, intuitiva e comunicativas das pessoas", têm provocado profundas mudanças em nossa sociedade, e não diferentemente, na comunidade científica. 
Nesta perspectiva de mudanças, Castells (2003a) parte do princípio de que pessoas, sociedades e processos, historicamente, é quem transforma a tecnologia. Este fato, segundo o autor, ocorre no momento em que nos apropriamos dela, modificando-a e experimentando-a.

No contraponto do entendimento destes recursos, buscamos em Lévy (1996, p. 46) a compreensão de que os recursos computacionais, como elementos do presente, não são mais do que fragmentos de peças e fusão de microequipamentos. Como elemento virtual, no entanto, tais tecnologias se tornam mais complexas e dinâmicas e se transformam em possibilidades, numa "turbulenta zona de trânsito para signos vetorizados".

O termo virtual adotado, diferentemente de como se pensa, não se opõe ao real, ao tangível, mas ao atual, ao momentâneo. Etnologicamente, "virtual" apresenta derivação do latim medieval virtualis, e significa força, potência.

O computador, na perspectiva virtual, deixa de ser compreendido apenas como um conjunto de componentes de soft e hardware, e se apresenta, constitutivamente, como um meio de comunicação centrado nos fluxos de informação.

No processo de evolução dos computadores, tais recursos ganharam interfaces mais complexas e humanizadas, fato ocorrido a partir da antiga Máquina de Turing ${ }^{3}$, que transformou o conceito de máquina meramente física e se transformou em um dispositivo teórico, uma ferramenta intelectual que processa signos (SANTAELLA, 2005).

Se, como apresenta esta autora, não há pensamento sem signos, por meio da grande capacidade de processamento de dados dos computadores modernos em captar, traduzir e transformar signos, o homem, como apresenta (CASTELLS, 2003a; 2003b), ao mesmo tempo, transforma e se transforma em tecnologias.

É na relação interpretativa de signos, sejam eles quantitativos ou não, que os softwares computacionais têm se apresentado como parceiros tanto da pesquisa, quanto do pesquisador. Ao viabilizar diferentes possibilidades de registro e de acompanhamento das relações entre os atores sociais, tais tecnologias têm auxiliado, significativamente, as fontes e formas de geração de indicadores sobre a sociedade, além de facilitar a formação de redes de bancos de dados.

A complexidade da sociedade moderna (ou pós-moderna, conforme abordagem de preferência do leitor), não concebe uma divisão tão evidente entre as abordagens quantitativas e qualitativas. É necessária, salvo particularidades, uma maior integração entre elas. A este respeito, é importante registrar a existência de fortes movimentos na área científica. Em especial, podemos

3 Máquina de Turing, também conhecida como máquina universal, desenvolvida pelo matemático britânico Alan Turing, no início da década de 1930. 
apresentar os procedimentos metodológicos da análise de conteúdo, aqui exemplificada em Bazeley (2007), Franco (2008), Richards, (2006), Bardin (2010); a pesquisa-ação, na linha francófona, a partir de Barbier (2007), Dione (2007), Moran (2009), Thiollent (2005); a fenomenologia, na perspectiva brasileira, a partir de Bicudo (2010), Barreto (2009), dentre outros.

O processo de desenvolvimento de programas computacionais para atendimento a esta tendência mista (quanti-quali), é bem recente, haja vista que os primeiros softwares de apoio às pesquisas de cunho qualitativo, de acordo com Teixeira (2009), surgiram, apenas a partir da década de 1980, momento em que houve um crescimento significativo desta modalidade de pesquisa em todo o mundo.

Organizados sob a sigla de Computer Aided Qualitative Data Analisys Softwares (CAQDAS), os softwares de análise qualitativa, inicialmente, se prestavam, de acordo com Baumgarten, Teixeira e Lima (2007), à análise de dados não numéricos, tendo como base a estrutura lógica do processamento estatístico de dados quantitativos.

Embora bastante eficazes quanto ao propósito das pesquisas qualitativas atuais, os softwares existentes no mercado (Atlas.TI, Max QDA, NVivo, dentre outros) não se caracterizam como softwares colaborativos, pois cada pesquisador, além de necessitar de uma licença individual, encarecendo os custos da pesquisa, deve concluir a sua etapa de análise para, só então, fazer o envio do seu arquivo a outro pesquisador ou equipe de pesquisa, fragmentando e retardando o processo (NERI DE SOUZA; COSTA; MOREIRA, 2011a).

Com a proposta de integração entre as pesquisas mistas em ambiente colaborativo distribuído, seguindo a tendência mundial de softwares acessíveis na Web social, conforme apresentam Neri de Souza, Costa e Moreira (2011b), o Centro de Investigação Didática e Tecnologia na Formação de Formadores do Departamento de Educação da Universidade de Aveiro, Portugal, lançou, em junho de 2011, o software WebQDA.

Segundo os autores, o software "auxilia os investigadores desde a fase da recolha de dados, até à fase da escrita das conclusões. Sendo um software de acesso online, cada projeto pode ser compartilhado por vários investigadores que sejam convidados e aos quais sejam atribuídos privilégios de acesso à plataforma" (p. 2). A proposta do software é proporcionar ao investigador, mesmo trabalhando individualmente, o acesso ao seu projeto via Web, e não somente por meio do programa instalado no computador.

Se, como apresenta Castells (2003b), mais do que tecnologia, a rede mundial de computadores, internet, se constitui como "tecido de nossas vidas" pelas 
infinitas possibilidades comunicacionais capazes de transformar a sociedade, denominada por ele de sociedade em rede, a possibilidade de integração entre pesquisas, numa perspectiva colaborativa e distribuída em rede, a nosso ver, se caracteriza como um caminho bastante fecundo.

Os tópicos seguintes buscam explorar os dados recolhidos no âmbito da investigação a fim de apresentar os resultados da pesquisa, partindo de uma perspectiva de aproximações entre os aspectos procedimentais de investigação e recursos tecnológicos utilizados nesse processo.

\section{Opções metodológicas e caracterização dos contextos}

A fim de averiguar a qualidade da formação de recursos humanos e das pesquisas no Brasil, os programas de pós-graduação em nível de mestrado e doutorado (stricto sensu) são avaliados trienalmente pelo MEC que atribui, como resultado de avaliação, notas no intervalo de 1 a 7 , sendo considerados de excelência os programas com notas iguais ou superiores a 5, eleitos nesta pesquisa como grupo de interesse.

As 19 Instituições de Ensino Superior (IES) pré-selecionadas foram: Pontifícia Universidade Católica do Rio de Janeiro (PUC-RIO); Universidade Estadual do Rio de Janeiro (UERJ); Universidade Federal de Minas Gerais (UFMG); Pontifícia Universidade Católica do Rio Grande do Sul (PUC-RS); Universidade Federal Fluminense (UFF); Universidade do Vale do Rio dos Sinos (UNISINOS); Universidade de São Paulo (USP); Universidade Federal do Espírito Santo (UFES); Universidade Federal de Goiás (UFG); Universidade Federal de Pelotas (UFPEL); Universidade Federal do Paraná (UFPR); Universidade Federal do Rio Grande do Sul (UFRGS); Universidade Federal do Rio de Janeiro (UFRJ); Universidade Federal do Rio Grande do Norte (UFRN); Universidade Federal de São Carlos (UFSCAR); Universidade Federal de Uberlândia (UFU), Universidade Estadual Paulista Júlio de Mesquita Filho, Câmpus de Marília (UNESP/MAR); Universidade Estadual de Campinas (UNICAMP); Universidade Metodista de Piracicaba (UNIMEP).

$\mathrm{Na}$ Figura 1 são mostradas as regiões do Brasil nas quais os programas de mestrado e doutorado, conforme critério de seleção apresentado, estão distribuídos.

Optou-se por uma amostragem estatisticamente significativa de docentes investigadores pertencentes ao quadro permanente das instituições selecionadas para que fosse possível apresentar um panorama mais assertivo da realidade a ser percebida. Nesse sentido, participaram da amostragem deste estudo 95 
docentes, de 10 das 17 IES cujos programas foram considerados de excelência, representando percentual acima de $15 \%$ do universo estatístico, dados que atingiram nível de confiança de $94 \%$ e margem de erro de $8 \%$. As IES selecionadas no âmbito da investigação foram: UFG, UFMG, UFRN, UFSCAR, UNESP/MAR, UNIMEP, UNICAMP, UNISINOS, USP e UFU.

\section{Figura 1 - programas de pós-graduação stricto sensu em educação no Brasil com notas iguais ou superiores a cinco}

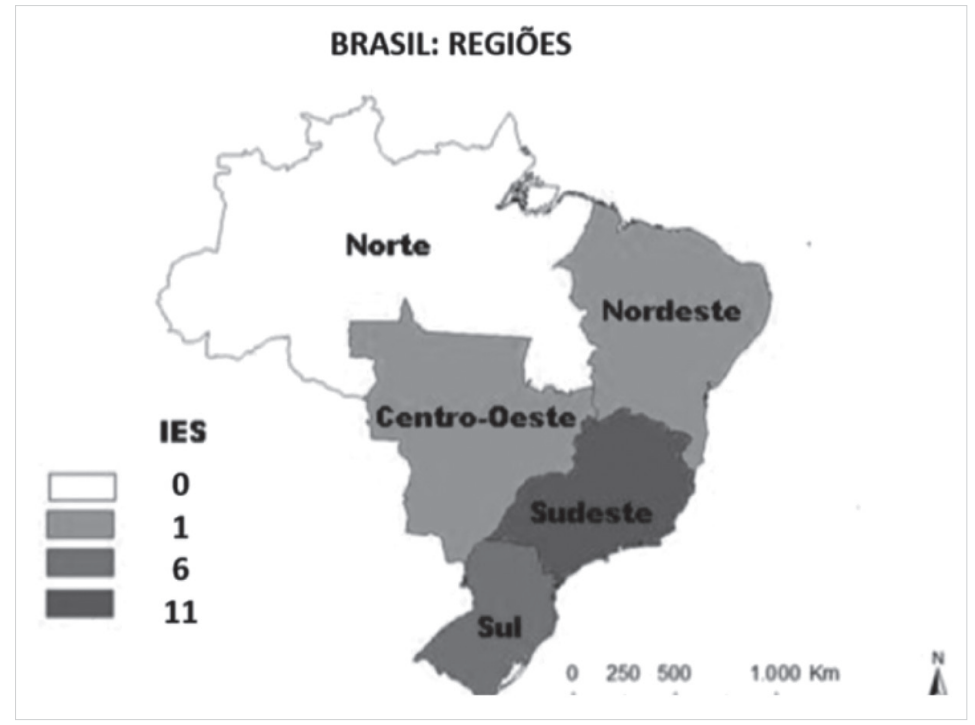

Fonte: Dados da pesquisa.

Utilizou-se como instrumento de recolha de dados um questionário contendo questões abertas e fechadas, aplicado em formato eletrônico. O questionário, contendo 30 questões, foi dividido em quatro partes, a saber: (1) conhecimento informático; (2) concepções e usos em metodologias da pesquisa; (3) nível de utilização de recursos informáticos para pesquisa; (4) caracterização da amostra.

Os dados foram coletados no período de 01 de agosto a 15 de setembro de 2012. Julgamos importante ressaltar que, embora o número de respondentes tenha atingido o quantitativo almejado, houve grandes dificuldades, devido à greve dos docentes das instituições federais de ensino, fator que ampliou bastante o período de aplicação e recolha. 
A base de análise partiu da abordagem quantitativa e estudos exploratórios, a partir de multiplicidades de dimensões de conteúdos presentes nos posicionamentos e ideias dos sujeitos pesquisados e dos variados dados coletados.

Como orientação dos procedimentos metodológicos, buscou-se na literatura as bases e fundamentos epistemológicos acerca da temática em voga, bem como documentos oficiais nos órgãos brasileiros para fundamentação política.

Como suporte a análise dos dados quantitativos utilizou-se o software estatístico SPSS, versão 20.

\section{Caracterização do perfil dos docentes investigadores e sua relação com as novas tecnologias}

Na perspectiva apresentada por Castells (2003b) de transformações multilaterais do homem em sua relação com as tecnologias, busca-se, a partir dos dados expostos, identificar e analisar tais perspectivas com o perfil dos docentes em estudo em seus processos de investigação.

A etapa de recolha de dados deste estudo se fundamentou na aplicação de questionários, enviados aos docentes de 10 das 17 instituições com conceito 5 ou mais, conforme exposto, no período estipulado. Os docentes investigadores que responderam ao questionário integravam as Instituições de Ensino Superior (IES), conforme dados apresentados no Gráfico 1.

\section{Gráfico 1 - Instituições de origem e número de docentes inquiridos na pesquisa}

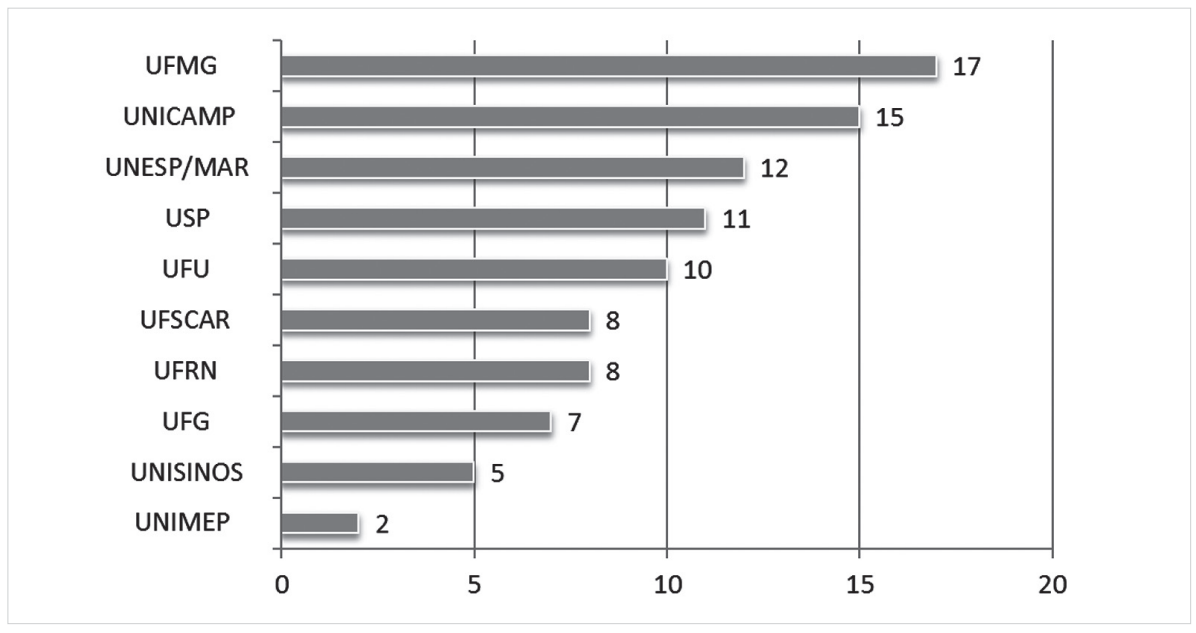

Fonte: Dados da pesquisa. 
Destaca-se que as instituições que mais colaboraram, em termos de participação, proporcionalmente ao número de professores do programa, foram: UFG (38,9\%), UNESP/Marília (33,3\%), UNISINOS (31,3\%), UFMG (28,8\%), UFU (27,8\%), UFSCar (27,6\%), UFRN (16,7\%), UNICAMP (16,3\%), UNIMEP $(14,3 \%)$ e USP $(14,1 \%)$. O número total de docentes que responderam ao questionário foi de 95.

Das 10 IES participantes da pesquisa, 5 (50\%) são instituições públicas federais; 3 (30\%), instituições públicas estaduais; e 2 (20\%) são instituições privadas. Do total das 19 IES brasileiras com nota 5 ou mais, $12(63,2 \%)$ possuem nota 5, $4(21,1 \%)$ possuem nota 6 e $3(15,8 \%)$ possuem nota 7 . Em termos de proporção das instituições, participaram da pesquisa $58,3 \%$ das IES com conceito $5,50,0 \%$ das IES com conceito $6 \mathrm{e}$, por fim, 33,3\% das IES com conceito 7 .

Com relação à formação superior dos docentes investigadores em nível de graduação, 31,4\% são de Pedagogia; 14,3\% de Psicologia; 7,6\% de Ciências Sociais; com 5,7\% apresentam-se as áreas de Biologia, Filosofia e História; com 2,9\%, as áreas de Matemática, Física, Educação Física e Letras; com 1,9\%, Comunicação Social e Informática. As demais áreas de formação, juntas, apresentaram frequência única e totalizaram os $10,5 \%$ restantes.

No Brasil há três modalidades ou graus de ensino em nível superior, sendo licenciatura, bacharelado e tecnólogo (BRASIL, 2010). A modalidade tecnólogo é recente e visa a atender, devido à natureza e menor tempo de formação, a um público ligado ao mercado de trabalho mais emergente, a fim de desenvolver competências técnicas. Diferentemente de outros países, toma-se aqui o exemplo de Portugal, os cursos de licenciatura são direcionados para formação de professores para atuarem na educação básica, enquanto os cursos de bacharelados são mais generalistas, com formação graduada completa voltada ao desenvolvimento científico e humanístico (BRASIL, 2010). Em Portugal, em conformidade com o Processo de Bolonha (Decreto-Lei $\mathrm{n}$. $74 / 2006)^{4}$, introduzido a partir de 2006, a licenciatura corresponde ao primeiro ciclo do ensino superior, com duração de três anos e, em casos especiais, de quatro anos. $\mathrm{O}$ bacharelado (ou bacharelato) correspondia a uma formação mais rápida, ofertada anteriormente ao Processo de Bolonha, portanto, não mais conferida pelas IES portuguesas.

Ressalta-se que, nas diferentes formações superiores dos docentes em pesquisa, embora os dados mostrem concentração na área de licenciatura

4 Ressalta-se que, além do decreto geral, para a formação de professores há um decreto específico, ou seja, o Decreto-Lei n. 43/2007. 
$(92,6 \%)$, sete $(7,4 \%)$ são oriundos do bacharelado, sendo 2 em Enfermagem; 2 em Engenharia; 1 em Química; 1 em Informática; e 1 em Administração Pública. A questão a ser observada é que, historicamente, há uma tendência no Brasil dos concursos públicos na área de educação privilegiarem docentes com formação na área de licenciatura, fator que justifica tal ênfase de análise.

No que diz respeito à idade dos docentes participantes da pesquisa, o Gráfico 2 mostra uma concentração maior na faixa etária de 41 a 60 anos, representando $76,5 \%$ de toda a amostragem, com média de 51,5 anos e desvio padrão de 8,84 .

\section{Gráfico 2 - Distribuição das idades dos docentes inquiridos}

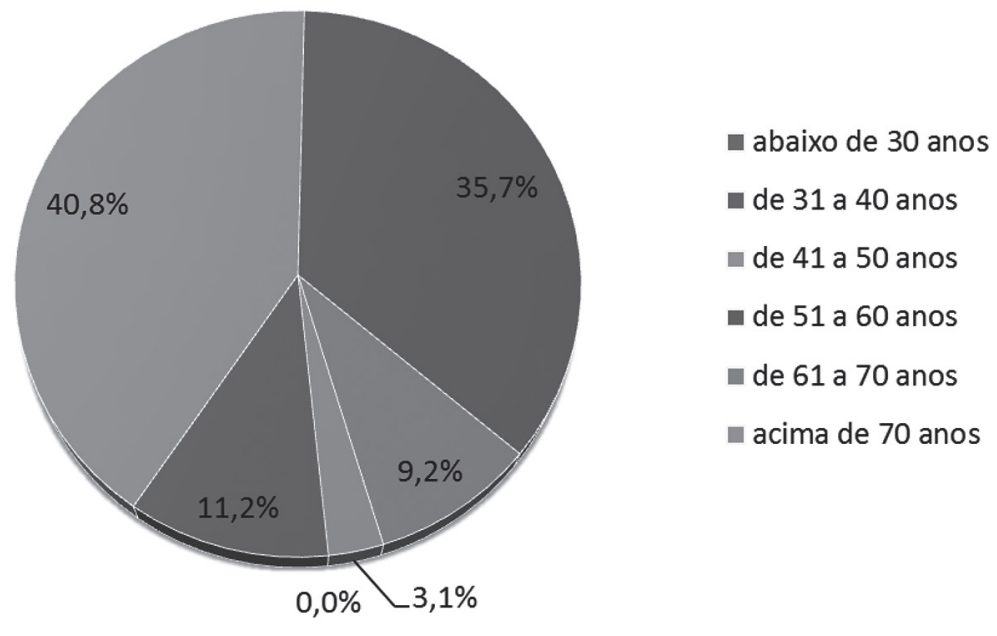

Fonte: Dados da pesquisa.

Em termos de distribuição, 11,2\% dos docentes apresentam perfil jovem (40 anos ou menos), com cinco ou menos anos de experiência em programas de mestrado e/ou doutorado (35,2\%); 40,8\% apresentam perfil mediano de idade (41 a 50 anos), com intervalo de docência entre 6 a 10 anos (15,4\%); e, do total dos docentes inquiridos, $44,9 \%$ apresentam perfil sênior em idade (51 anos ou mais) e com tempo de experiência acima de 10 anos (49,5\%).

Ainda com relação à formação superior, ao serem questionados sobre há quanto tempo fizeram sua primeira graduação, os docentes apresentaram as seguintes informações, representadas no Gráfico 3. 


\section{Gráfico 3 - Tempo, em anos, de formação superior na primeira graduação}

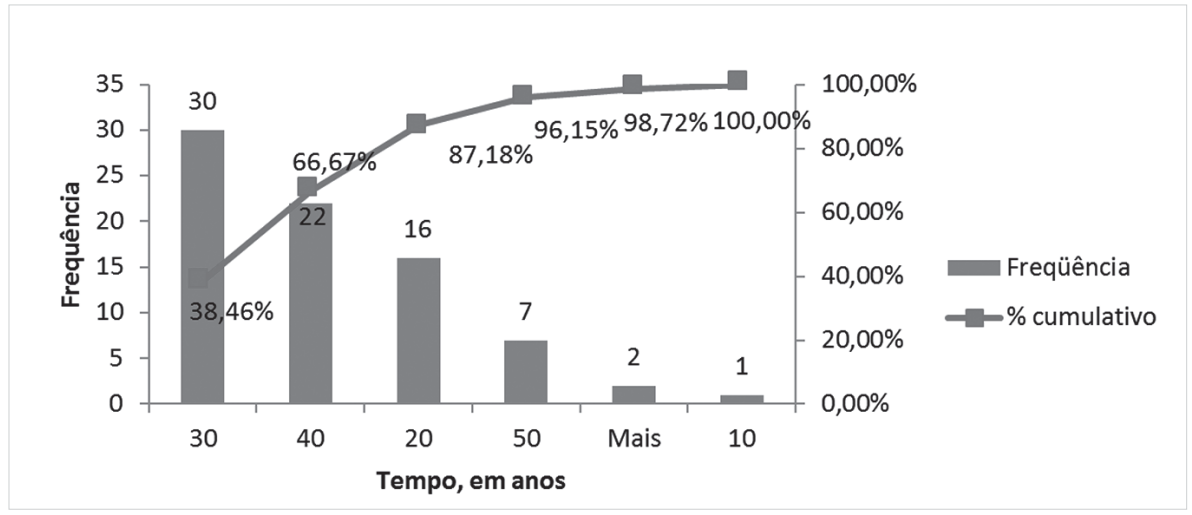

Fonte: Dados da pesquisa.

Observa-se, por meio do Gráfico de Pareto, que a maioria dos investigadores concluiu sua primeira graduação no intervalo de 20 a 40 anos atrás $(87,2 \%)$, com média de 27,15 anos () e desvio padrão de 1,17 (). Complementa-se que os dados contidos no eixo das abscissas refletem os seguintes intervalos de valores: menor que 10 anos; de 11 a 20 anos; de 21 a 30 anos; de 31 a 40 anos; de 51 a 50 anos; mais que 50 anos.

Fazendo um paralelo entre as idades da formação acadêmica de pós-graduação dos pesquisadores docentes em estudo, tem-se média de idade para formação de mestrado em 31,6 anos ( e doutorado de 39,1 anos de idade. (Na Tabela 1, observa-se que há certa harmonização de transição de idade entre a graduação e pós-graduação (mestrado e doutorado) - processo de translação dos dados em idades.

A faixa etária, já expressa, porém, agora com intervalos mais precisos, continua concentrada na faixa etária de 20 a 30 anos, com representatividade de $89,7 \%$ de toda amostra. No mestrado, com média de 31,6 anos de idade e desvio padrão de , a faixa etária encontra-se no intervalo de 26 a 35 anos, com concentração de $72,9 \%$ dos dados. Por fim, agora, pois, mais disperso, o intervalo de idade dos docentes pesquisadores nos programas de doutorado em educação no Brasil apresenta-se no intervalo de 31 a 45 anos ( anos e ).

Com o propósito de verificar se existe ou não correlação entre as variáveis relativas à idade dos docentes em estudo em seus diferentes processos de formação, apresenta-se, nos Gráficos 4, 5 e 6, os dados para a graduação, o mestrado e doutoramento. 
Tabela 1 - Intervalo de idade na formação, graduação e pós-graduação

\begin{tabular}{c|c|c|c|c|c|c}
\hline $\begin{array}{c}\text { INTERVALO DE } \\
\text { IDADE }\end{array}$ & GRADUAÇÃO & $\%$ & MESTRADO & $\%$ & DOUTORADO & $\%$ \\
\hline 20 anos ou menos & 7 & $9,0 \%$ & 0 & $0,0 \%$ & 0 & $0,0 \%$ \\
\hline de 20 a 25 anos & 61 & $78,2 \%$ & 4 & $5,7 \%$ & 0 & $0,0 \%$ \\
\hline de 26 a 30 anos & 9 & $11,5 \%$ & 30 & $42,9 \%$ & 4 & $4,5 \%$ \\
\hline de 31 a 35 anos & 0 & $0,0 \%$ & 21 & $30,0 \%$ & 23 & $26,1 \%$ \\
\hline de 36 a 40 anos & 1 & $1,3 \%$ & 11 & $15,7 \%$ & 24 & $27,3 \%$ \\
\hline de 41 a 45 anos & 0 & $0,0 \%$ & 3 & $4,3 \%$ & 22 & $25,0 \%$ \\
\hline de 46 a 50 anos & 0 & $0,0 \%$ & 1 & $1,4 \%$ & 11 & $12,5 \%$ \\
\hline de 51 a 55 anos & 0 & $0,0 \%$ & 0 & $0,0 \%$ & 3 & $3,4 \%$ \\
\hline de 56 a 60 anos & 0 & $0,0 \%$ & 0 & $0,0 \%$ & 1 & $1,1 \%$ \\
\hline acima de 60 anos & 0 & $0,0 \%$ & 0 & 0 & 0 & 0 \\
\hline N & 78 & $100,0 \%$ & 70 & $100,0 \%$ & 88 & $100,0 \%$ \\
\hline
\end{tabular}

Fonte: Dados da pesquisa.

\section{Gráfico 4 - Correlação entre idade e ano de conclusão do programa de graduação}

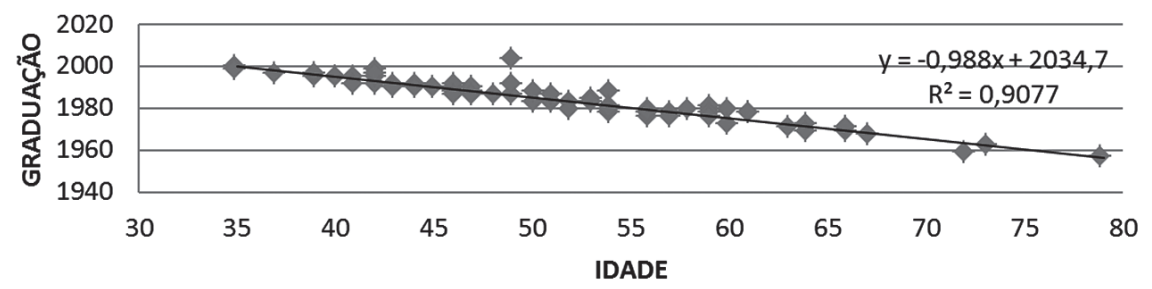

Fonte: Dados da pesquisa.

Gráfico 5 - Correlação entre idade e ano de conclusão do programa de mestrado

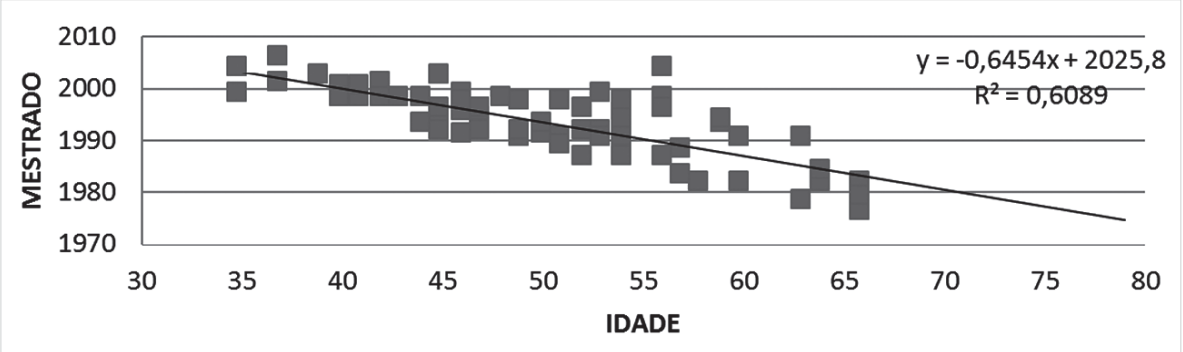

Fonte: Dados da pesquisa. 


\section{Gráfico 6 - Correlação entre idade e ano de conclusão do programa de doutorado}

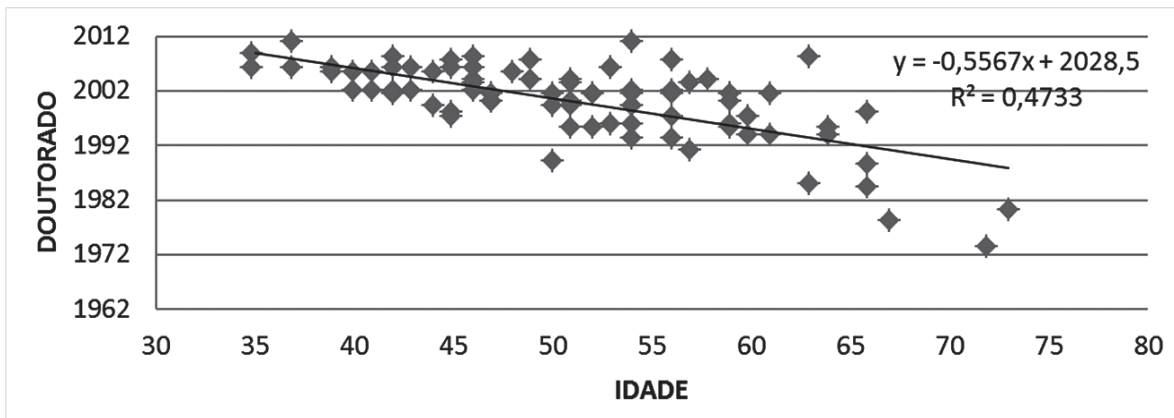

Fonte: Dados da pesquisa.

No Gráfico 4, a relação entre idade e ano de graduação apresenta pontos com certa aderência (ou aproximação) à reta, denominada de curva de tendência. No Gráfico 5, idade e ano do mestrado, esta aderência não é tão forte, reduzindo-se ainda mais no Gráfico 6, idade e ano de doutorado. Os gráficos e os dados neles contidos nos subsidiam com informações para inferências estatísticas.

Quanto à idade dos docentes e sua formação em nível de graduação, pode-se inferir forte correlação, com indicador de 0,953 (), ou seja, com coeficiente de determinação de $90,77 \%$ (). Ao avançar no processo de formação, a correlação perde força sequentemente para o mestrado (0) e para o doutorado (). Isto significa que o grau de doutor entre os professores na área de educação no Brasil foi obtido numa dispersão de idade muito maior que os graus precedentes.

Dentro de uma perspectiva de prática docente, a experiência dos inquiridos em termos de tempo, em anos, dedicados nos diferentes níveis educacionais encontra-se expressa na Tabela 2. Ao objetivar compreender o perfil dos investigadores da área educacional, tal conhecimento no âmbito do ensino é parte essencial deste construto.

A partir do agrupamento dos dados de modalidades por blocos (Educação Básica e Ensino Superior), observa-se que, dos docentes que atuam em programas de mestrado e doutorado em educação ( anos e ), 30,8\% têm experiência com a Educação Básica. Deste montante, 16,3\% disseram ter atuado na Educação Infantil ( anos e ); 24,2\%, nos anos iniciais do Ensino Fundamental: Ensino Fundamental I ( anos e ); 26,8\%, nos anos finais do Ensino Fundamental: Ensino Fundamental II ( anos e ); e 32,7\%, disseram ter experiência com o Ensino Médio ( anos e ). 
Tabela 2 - Tempo de docência nas fases ou etapas de ensino

\begin{tabular}{|c|c|c|c|c|c|c|c|}
\hline & MODALIDADES & $\begin{array}{c}\text { Menos } \\
\text { de } 1 \text { ano }\end{array}$ & $\begin{array}{l}\text { De } 1 \text { a } \\
5 \text { anos }\end{array}$ & $\begin{array}{c}\text { De } 6 \text { a } \\
10 \text { anos }\end{array}$ & $\begin{array}{c}\text { De } 11 \text { a } 15 \\
\text { anos }\end{array}$ & $\begin{array}{c}\text { Acima } \\
\text { de } 16 \\
\text { anos } \\
\end{array}$ & TOTAL \\
\hline \multirow{4}{*}{ 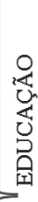 } & Educação Infantil & 5 & 12 & 5 & 0 & 3 & 25 \\
\hline & Ensino Fundamental I & 10 & 17 & 7 & 0 & 3 & 37 \\
\hline & Ensino Fundamental II & 8 & 18 & 9 & 3 & 3 & 41 \\
\hline & Ensino Médio & 9 & 20 & 10 & 3 & 8 & 50 \\
\hline \multirow{5}{*}{ 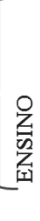 } & Graduação & 1 & 3 & 12 & 19 & 56 & 91 \\
\hline & Pós-Graduação lato sensu & 1 & 27 & 19 & 10 & 15 & 72 \\
\hline & Pós-Graduação stricto sensu & 4 & 26 & 15 & 24 & 22 & 91 \\
\hline & Pós-Graduação em Educação & 4 & 28 & 14 & 30 & 15 & 91 \\
\hline & TOTAL & 42 & 151 & 91 & 89 & 125 & 498 \\
\hline
\end{tabular}

Fonte: Dados da pesquisa.

Em se tratando de programas de Pós-Graduação em Educação, tal experiência e vivência se apresentam como importantes tanto para o acompanhamento das problemáticas, níveis de escolaridade e ao grupo quanto pela sensibilidade adquirida para condução dos processos de orientação e investigação.

Para avaliar os conhecimentos em informática do grupo inquerido, foram selecionadas 6 questões que buscaram compreender o nível técnico e operacional do usuário, sendo elas relacionadas a: como aprendeu a operar o computador (1); níveis de utilização de recursos, serviços, linguagens e ferramentas computacionais (2); acesso a computador e internet (3); tempo médio, em horas por semana, de uso do computador (4) e internet (5); autoavaliação, em escala Likert, sobre o conhecimento em informática na condição de utilizador (6).

Ao solicitar uma autoavaliação sobre o uso de recursos computacionais, na escala de 1 (pouco) e 5 (muito), verificou-se, com maior representação, uma concentração de $54,5 \%$ na escala 3 , seguida de $24,3 \%$ na escala 4 e $8,9 \%$ na escala 2. Como se suspeitava, os dados mostram maiores indicadores na escala para os docentes mais jovens, principalmente aqueles com idades abaixo de 50 anos, embora isso não indique que docentes com idades mais avançadas não tenham domínio de recursos computacionais e nem que os mais jovens sejam experts em seu uso.

Sobre como aprenderam a operar o computador, 59,2\% disseram que foi a partir da exploração autônoma das suas ferramentas e funções; $24,5 \%$, com auxílio de familiares e/ou pessoas próximas; $16,3 \%$, por meio de cursos de 
informática; e, apenas, um docente investigador (1,02\%) disse não saber utilizar o computador.

Quanto ao acesso a computador e internet, foi solicitado que indicassem o posicionamento frente a um conjunto de afirmações. Os resultados encontram-se na Tabela 3.

\section{Tabela 3 - Acesso ao computador e internet}

\begin{tabular}{|c|c|c|c|}
\hline Proposições & Sim & Não & Frequência \\
\hline Tenho meu próprio computador portátil (laptop ou notebook) & $92,9 \%(91)$ & $7,1 \%(7)$ & $100 \%(98)$ \\
\hline $\begin{array}{l}\text { A instituição disponibiliza um computador portátil para meu } \\
\text { uso }\end{array}$ & $50,0 \%(47)$ & $50,0 \%(47)$ & $100 \%(94)$ \\
\hline Tenho meu próprio computador fixo (desktop ou gabinete) & $83,0 \%(78)$ & $17,0 \%(16)$ & $100 \%(94)$ \\
\hline A instituição disponibiliza um computador fixo para meu uso & $91,9 \%(91)$ & $8,1 \%(8)$ & $100 \%(99)$ \\
\hline Tenho acesso próprio à internet sem fio & $91,7 \%(88)$ & $8,3 \%(8)$ & $100 \%(96)$ \\
\hline Tenho acesso próprio à internet com fío & $85,3 \%(77)$ & $14,7 \%(13)$ & $100 \%(90)$ \\
\hline Tenho acesso à internet sem fio na instituição & $85,3 \%(81)$ & $14,7 \%(14)$ & $100 \%(95)$ \\
\hline Tenho acesso à internet com fío na instituição & $96,8 \%(92)$ & $3,2 \%(3)$ & $100 \%(95)$ \\
\hline $\begin{array}{l}\text { A instituição tem laboratório de informática com } \\
\text { computadores com acesso à internet }\end{array}$ & $98,0 \%(97)$ & $2,0 \%(2)$ & $100 \%$ (99) \\
\hline
\end{tabular}

Fonte: Dados da pesquisa.

Em linhas gerais, exceto na disponibilização de computadores portáteis, os dados mostram sinalização positiva em relação à aquisição pessoal e institucional de recursos de acesso e equipamentos informáticos.

Sobre a utilização de recursos, serviços, linguagens e ferramentas computacionais, 79,2\% dos docentes disseram utilizar, em concordância total, o computador como apoio e suporte à pesquisa e 53,5\%, como apoio ao processo pedagógico, enquanto $78,2 \%$ utilizam tais recursos em outros afazeres em seu dia a dia. É, também, considerável a utilização de computadores e acesso à internet por parte dos docentes, cuja utilização ultrapassa, em maioria, carga de 20 horas semanais $(67,3 \%$ e $50,5 \%$, respectivamente), ficando em nível insignificante em relação à amostragem, cargas inferiores há 10 horas semanais destes recursos combinados.

\section{Aproximações na relação entre novas tecnologias e sua utilização nos diferentes processos de pesquisa}

Com intenção de relacionar, pensadas neste espaço como aproximações, o uso dos recursos tecnológicos e sua utilização nos processos de pesquisa, inqueriu-se os docentes investigadores no sentido de obter informações acerca 
de estrutura, referências, abordagens, procedimentos, epistemologia, bem como compreender os posicionamentos no âmbito das investigações.

Acerca das bases eletrônicas de dados para referências bibliográficas, tendo o pesquisador a possibilidade de marcar até 9 opções, podendo, em uma delas, indicar outras não expostas, 93,1\% ( $\mathrm{n}=94)$ disseram utilizar a plataforma eletrônica Scielo; 91,1\% ( $\mathrm{n}=92)$, o Portal da Capes 5 ; 86,1\% ( $\mathrm{n}=93)$, Banco de Teses; 68,3\% ( $\mathrm{n}=94)$ Google Acadêmico; 41,6\% ( $\mathrm{n}=91)$ Google Books; 24,8\% (n=94) Scorpus; 10,9\% (n=93) Digital Bibliography \& Library Project (DBLP). Dos 25 que marcaram a opção “outro", cada uma das seguintes bases de dados foi indicada duas vezes ( $8 \%$ cada), sendo elas Eric; Sibi-USP BVS-PSI; e SAGE. Ressalta-se que apenas 3\% disseram não utilizar nenhuma base de consulta eletrônica de dados.

Ao serem questionados sobre os recursos tecnológicos mais utilizados na recolha de dados primários, podendo marcar até seis itens, sendo "outro" um deles, o gravador digital foi o instrumento mais indicado, com $67,0 \%(\mathrm{n}=63)$, seguido do questionário ou formulário digital, $59,6 \%(\mathrm{n}=56)$; máquina fotográfica $52,1 \%(n=49)$; câmera filmadora, $45,7 \%(n=43)$; dados e relatórios internacionais, 43,6\% $(n=41)$; e 2,1\% $(n=2)$ disseram não utilizar. $O$ item "outros" apresenta grande dispersão de recursos, destacando scanner 2,1\% $(\mathrm{n}=2)$ e reciclagem de dados $2,1 \%(\mathrm{n}=2)$.

Além das informações sobre o uso de recursos tecnológicos nas diferentes fases de pesquisa, adentrando na especificidade do software, buscou-se o cruzamento de dados com a especificidade dos recursos informáticos em termos de aproximações. Observa-se, neste sentido, que a Tabela 3 apresenta variadas informações passíveis de análises.

$\mathrm{Na}$ fase de revisão bibliográfica e/ou documental, partindo de análise cruzada da tabela e podendo os inqueridos marcar mais de uma opção, os investigadores apresentaram, com maior ênfase, a utilização de computadores/ terminais com 76,8\% (n=97). Faz-se necessário ressaltar que tais tecnologias destacam-se em todas as fases do processo de investigação. Na sequência da revisão bibliográfica e/ou documental, segue a utilização de banco de dados, $35,4 \%(n=35)$ e rede de transmissão de dados, $27,3 \%(n=27)$.

Com relação ao momento de recolha de dados, como já anunciado, destaca-se a utilização bancos de dados com equipamentos analógicos ou digitais com 26,1\% ( $\mathrm{n}=49)$; banco de dados, 19,7\% ( $\mathrm{n}=37)$; e equipamentos analógicos e digitais com $17,6 \%(n=33)$.

5 O Portal de Periódico CAPES (http://www.periodicos.capes.gov.br/) dá acesso ao pesquisador no Brasil à maioria das publicações da ISI Web of Knowledge, entre outras bases de dados. 
$\mathrm{Na}$ organização de dados, a sequência de utilização dos recursos obedece à seguinte ordem: computadores e terminais, com 31,0\% $(\mathrm{n}=53)$; software adquirido, $19,3 \%(\mathrm{n}=33)$; e banco de dados, $17,5 \%(\mathrm{n}=30)$.

Dentro da mesma lógica de distribuição e estruturação, na fase de tratamento de dados, tem-se: computadores e terminais, $31,1 \%(\mathrm{n}=46)$; software adquirido, 25,7\% ( $\mathrm{n}=38)$; e banco de dados com $11,5 \%(\mathrm{n}=17)$.

$\mathrm{Na}$ última fase inquerida, análise de dados, os recursos e a sequência de utilização foram exatamente a mesma da fase anterior, com variação apenas na frequência que, consecutivamente, foram: computadores $33,8 \%(\mathrm{n}=46)$; software 21,3\% ( $\mathrm{n}=29)$; e banco de dados $14,0 \%(\mathrm{n}=19)$. Embora se observe recorrência de outros recursos nos diferentes processos de investigação, estes, porém, não obtiveram destaque para análise.

Analisemos os dados a partir de outra perspectiva, agora, por representatividade total, a partir da distribuição, mostrada na Tabela 4.

\section{Tabela 4 - Recursos computacionais utilizados, por representação global, nas diferentes fases do processo investigativo}

\begin{tabular}{|l|c|c|}
\hline \multicolumn{1}{|c|}{ OPÇÕES } & Fr & $\%$ \\
\hline Computadores/ Terminais & 270 & $33,0 \%$ \\
\hline Banco de Dados & 138 & $16,9 \%$ \\
\hline Software adquirido & 137 & $16,7 \%$ \\
\hline Rede de computadores & 100 & $12,2 \%$ \\
\hline Equipamentos analógicos ou digitais & 80 & $9,8 \%$ \\
\hline Rede de transmissão de dados & 38 & $4,6 \%$ \\
\hline Software desenvolvido (modelado) & 29 & $3,5 \%$ \\
\hline Laboratório de informática & 26 & $3,2 \%$ \\
\hline TOTAL & 818 & $100,0 \%$ \\
\hline
\end{tabular}

Fonte: Dados da pesquisa.

Sobre a especificidade do uso de software nas investigações, esta foi a questão que representou o maior nível de abstenção (41\%). Os dados coletados subsidiam informações sobre a crença de que tais recursos ainda não são percebidos por parte dos inquiridos como instrumento de apoio e suporte 
à investigação, embora muitos se posicionem de forma contrária. Para tanto, faz-se necessário avançar nos processo de análise.

Dos que disseram utilizar software $(59,9 \%)$, tem-se maior representatividade, em termos de frequência, os softwares de análise quantitativa $(41,1 \%)$, seguidos de software de análise qualitativa $(39,4 \%)$ e, por fim, o uso de software de referência $(15,5 \%)$, como mostra o Gráfico 7.

\section{Gráfico 7 - Uso de software na organização, tratamento e análise dos dados}

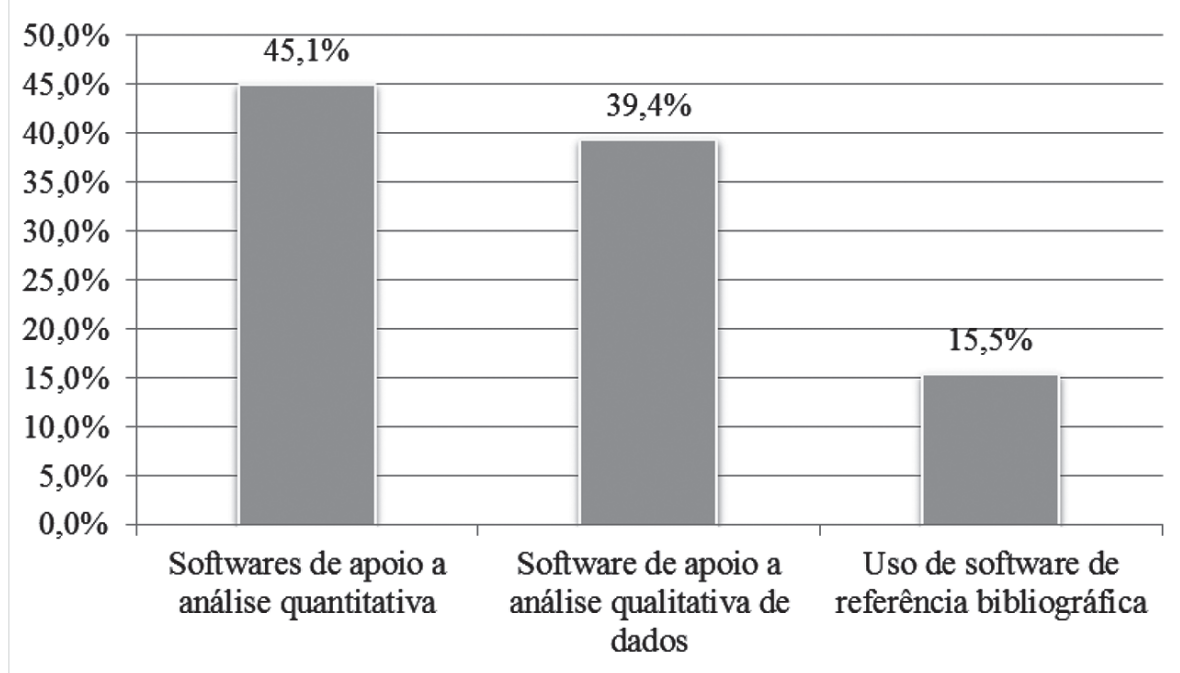

Os exemplos, como subsídio, foram assim organizados: software de referência bibliográfica: Endnote, LaTEX e/ou outros; software de apoio à análise de dados quantitativos: SPSS, Sphinx, R, Minitab, ALCESTE e/ou outros; e software de apoio à análise de dados qualitativos: WebQDA, Atlas.TI, Max QDA, NVivo e/ou outros. Na estrutura de "outros", os softwares citados foram: MS Excel (2), Word (2), Access (1), Vantage Point (1), Ucinet (1) e ScriptLattes (1).

Retomando a propositura de análise para as informações anunciadas, interessa-nos apresentar os seguintes dados, expostos na Tabela 5, no sentido de confrontar as informações contidas no Gráfico 7.

Tais informações contidas na Tabela 5, em primeira análise, são contraditórias no que diz respeito às do Gráfico 7 . Na condução desta ideia, é notório perceber que, por apresentar maior frequência de pesquisadores da área qua- 
litativa (52,5\%), seria, pois, razoável que os softwares mais utilizados fossem os que contemplassem esta área, o que, contudo, não ocorreu.

\section{Tabela 5 - Modalidade das pesquisas mais adotadas pelos docentes investigadores}

\begin{tabular}{|c|c|c|}
\hline MODALIDADE DE PESQUISA & $\mathrm{Fr}$ & $\%$ \\
\hline Pesquisas de cunho qualitativo & 53 & $52,5 \%$ \\
\hline Pesquisas de cunho quantitativo & 2 & $2,0 \%$ \\
\hline Pesquisas mistas (quanti-qualitativa) & 46 & $45,5 \%$ \\
\hline TOTAL & 101 & $100,0 \%$ \\
\hline
\end{tabular}

Fonte: Dados da pesquisa.

A questão, porém, está concentrada em três movimentos a serem atentados. O primeiro diz respeito ao alto índice de abstenção na resposta, fator que, decerto, tem um indicativo forte da não utilização dos recursos, tendo por base o volume de respostas nas demais questões do instrumento.

O segundo, o que nos dá maior consistência para inferências, é o fato de haver um número considerável de investigadores que se utilizam da modalidade mista de pesquisa. Supõe-se, neste sentido, que ao realizar pesquisas quali-quantitativas, no âmbito das análises dos dados, muitos busquem nos softwares "quanti" o apoio e suporte para tratamento e análises estatísticas.

Um terceiro fator, porém, não confirmado, seria considerar que parte das abstenções de respostas ao item em anunciado pudesse corresponder à fatia dos que investiguem na área qualitativa.

Um fator essencial de análise é que as contradições polares históricas existentes na relação entre as abordagens qualitativa e quantitativa apontadas por Lefevre e Lefevre (2005), mostram-se flexibilização frente ao número considerável de investigações de cunho misto, tendência potencializada com o surgimento de softwares de apoio a análises de dados.

De acordo com Neri de Souza, Costa e Moreira (2011a e 2011b), com o uso de softwares, as possibilidades de exploração dos dados se multiplicam, dando condições para que o investigador avance em seus processos de pesquisa e sistematizem suas análises, dando maior consistência e rigor à investigação, em muitos casos, além de potencializar, quando for o caso, os trabalhos colaborativos. 


\section{Considerações finais}

Embora os estudos tenham focado nos processos de pesquisa dos investigadores brasileiros, as atividades docentes no Brasil, diferentemente, por exemplo de Portugal, mesmo que as universidades gozem de autonomia em seus processos de ensino e de gestão, a tríade: ensino, pesquisa e extensão fazem parte das funções profissionais a serem exercidas e contempladas como garantidas na Constituição Federal do País, de 1988 (BRASIL, 1988), ratificadas pelas Leis de Diretrizes e Bases da Educação Nacional, de 1996 (BRASIL, 1996) e observadas em diversos documentos oficiais do MEC.

A indissociabilidade entre tais dimensões, Art. 207 da CF/1988, converge para que as atividades sejam, de certa forma, desempenhadas de maneira interligada, contínua e harmônica.

A articulação indissociável entre ensino e pesquisa reflete, na extensão, as dimensões educativa, cultural, política e científica integrantes da atuação universitária, que deve convergir em contribuições, preferencialmente, ao desenvolvimento em âmbito local, regional e nacional (FORPROEX, 2012).

Neste processo, ao perseguir, no âmbito do estudo, o perfil dos investigadores brasileiros, no recorte da área de educação, por conseguinte, tem-se um parâmetro, em termos de reflexo, do perfil de docência e das ações extensivas. De forma sintética, como resultado do estudo, chega-se a algumas considerações. Dentre elas destacam-se:

a) grande concentração de programas de mestrado e doutorado de excelência em educação na Região Sudeste do Brasil, representando 64,3\% do universo segmentado;

b) presença significativa de novos docentes nos respectivos programas de Mestrado em Educação (38,8\% com 5 ou menos anos de dedicação), sendo grande parte com idade igual ou inferior a 40 anos $(11,2 \%)$;

c) a expectativa confirmada acerca da formação, em nível de graduação, nas áreas de licenciatura $(92,6 \%)$;

d) que há forte correlação entre a idade dos docentes com o período de graduação, porém, não confirmada em relação ao processo de doutoramento, fator que suscita possíveis interseções entre parcela das diferentes gerações de doutores com formação em períodos de tempo próximos; 
e) a vivência prática/experiência profissional de parcela dos docentes inquiridos (30,8\%) na Educação Básica, apontada pelos autores como importantes nos processos de formação e condução das investigações;

f) dentro de perspectivas metodológicas, que, embora a maioria dos docentes trabalhe em uma linha mais qualitativa de investigação (52,5\%), há um grupo significativo valendo-se de pesquisa mista ou quali-quantitativa $(45,5 \%)$;

g) que quase a totalidade dos inquiridos utiliza-se de bases eletrônicas de dados (97,0\%); fazem uso de recursos tecnológicos como máquinas e equipamentos para recolha de dados primários (97,9\%); usam computadores $(33,0 \%)$, banco de dados $(16,9 \%)$ e softwares adquiridos $(16,7 \%)$;

h) na questão do uso de software, pela contradição de haver representação qualitativa do grupo e utilização concentrada nos softwares estatísticos, bem como o alto nível de abstenção na resposta da questão, consideramos que parte significativa dos inquiridos não fazem uso de tais recursos nos processos de análise de dados por desconhecerem estas ferramentas e/ou porque o número destacado da linha quantitativa se justificar pelos investigadores que adotam pesquisas mistas como procedimento de pesquisa.

Para finalizar, no presente artigo, buscou-se discutir, com base no perfil dos docentes de programas de pós-graduação stricto sensu em Educação, as relações entre os processos de pesquisa e as tecnologias computacionais no âmbito da investigação.

Concordamos com Henwood e Pidgeon (1992) quando dizem que os métodos não possuem valor em si, mas na medida em que respondem melhor ou pior à questão de investigação específica.

\section{Agradecimentos}

Ao Centro de Investigação em Didática e Tecnologia na Formação de Formadores (CIDTFF) da Universidade de Aveiro e à Universidade Federal de Goiás (UFG) que proporcionaram condições para o desenvolvimento do presente estudo. 


\section{Referências}

BARBIER, R. A pesquisa-ação. Brasília: Liber Livro, 2007.

BARDIN, L. Análise de conteúdo. 4. ed. Lisboa: Edições 70, 2010.

BARRETO, M. F. T. O tempo vivido pelo alfabetizando adulto nas aulas de matemática. Educar em Revista, Curitiba, v. 33, p. 187-203, 2009.

BAUMGARTEN, M.; TEIXEIRA, A. N.; LIMA, G. Sociedade e Conhecimento: novas tecnologias e desafios para a produção de conhecimento nas Ciências Sociais. Sociedade e Estado, Brasília, v. 22, n. 2, p. 401-433, maio/ago., 2007.

BAZELEY, P. Qualitative Data Analysis with NVivo. London: SAGE Publications, 2007.

BICUDO, M. A. V. Filosofia da educação matemática. Fenomenologia, concepções, possibilidades didático-pedagógicas. São Paulo: EdUNESP, 2010.

BRASIL. Constituição Federal: acompanhada das disposições anteriores, emendas constitucionais de revisão, índices sistemático e alfabéticoremissivo. São Paulo: Editora Oliveira Mendes, 1988.

BRASIL.MEC. Lei de Diretrizes e Bases da Educação Nacional. (Lei n. 9.394). Brasília-DF, 1996.

\section{. Referenciais Curriculares Nacionais dos Cursos de}

Bacharelado e Licenciatura/Secretaria de Educação Superior. Brasília: Secretaria de Educação Superior, 2010. Disponívem em: < http://emec.mec. gov.br/emec/educacao-superior/cursos $>$. Acesso em: 20 ag. 2013

CASTELLS, M. A galáxia da internet: reflexões sobre a internet, os negócios e a sociedade. Tradução de BORGES, M. L. X. D. A. Rio de Janeiro: Zahar, 2003a.

. Internet e sociedade em rede. In: MORAES, D. D. (Ed.). Por uma outra comunicação - mídia, mundialização cultural e poder. Rio de Janeiro: Record, 2003b. 
DENZIN, N. K.; LINCOLN, Y. S. Strategies of qualitative inquiry. 3. ed. Thousand Oaks: Sage, 2008.

DIONE, H. A pesquisa-ação para o desenvolvimento local. Brasília: Liber Livro, 2007.

FRANCO, M. L. P. B. Análise do conteúdo. Brasília: Liber Livro, 2008.

FORPROEX. Fórum de Pró-Reitores de Extensão das Universidades Públicas Brasileiras. Política nacional de extensão universitária. Manaus: FORPROEX, 2012.

HENWOOD, K. L.; PIDGEON, N. F. Qualitative research and psychological theorizing. British Journal of Psychology, London, v. 83, p. 97-111, 1992.

KENSKI, V. M. Educação e tecnologias: o novo ritmo da informação. Campinas: Papirus, 2007.

LEFEVRE, A. M.; LEFEVRE, F. Depoimentos e discursos: uma proposta de análise em pesquisa social. Brasília: Líber Livro, 2005.

LÉVY, P. O que é o virtual? São Paulo: Editora 34, 1996.

LIMA, T. C. S. L.; MIOTO, R. C. T. Procedimentos metodológicos na construção do conhecimento científico. Ver. Katál, Florianópolis, v. 10, n. esp, p. 37-45, 2007.

MILES, M. B.; HUBERMAN, A. M. Qualitative data analysis. 2. ed. London: Sage Publications, 1994.

MORAN, J. M. Desafios dos educadores com as tecnologias. In: MORAN, J. M. (Ed.). Educação 2009: as mais importantes tendências na visão dos mais importantes educadores. Curitiba: Multiverso, 2009.

NERI DE SOUZA, F.; COSTA, A. P.; MOREIRA, A. WebQDA: Análise de dados qualitativos suportada pelo software WebQDA. In: CONFERÊNCIA INTERNACIONAL DE TECNOLOGIA DE INFORMAÇÃO E COMUNICAÇÃO NA EDUCAÇÃO, 7., 2011, Braga-Portugal. [Anais...]. Braga-Portugal: Universidade do Minho, 2011a. 
NERI DE SOUZA, F.; COSTA, A. P.; MOREIRA, A. Análise de dados qualitativos suportada pelo software WebQDA. Challenges - 2011. Braga-Portugal: Universidade do Minho, 2011b.

RICHARDS, L. Handling qualitative data. London: Sage Publications, 2006.

SANTAELLA, L. Matrizes da linguagem e pensamento: sonora, visual e verbal - aplicações na hipermídia. São Paulo: Iluminuras, 2005.

SANTOS, B. S. Um discurso sobre as ciências. São Paulo: Cortez, 2009.

TEIXEIRA, A. N. A produção televisiva do crime violento na

modernidade tardia. 2009. Tese (Doutorado) - Universidade Federal do Rio Grande do Sul, Porto Alegre, RS, 2009.

THIOLlENT, M. J. M. Metodologia da pesquisa-ação. 14. ed. São Paulo: Cortez, 2005.

Ricardo Antonio Gonçalves Teixeira - Universidade Federal de Goiás Goiania | GO | Brasil. Contato: Professorricardoteixeira@gmail.com

Francislê Neri de Souza - Universidade de Aveiro Aveiro | Portugal. Contato: fns@ua.pt

Rui Marques Vieira - Universidade de Aveiro Aveiro | Portugal. Contato: rvieira@ua.pt

Artigo recebido em 31 de outubro de 2013 e aprovado em 7 de abril de 2013. 